\title{
Incidence of Acute Disseminated Encephalomyelitis in China: First National Survey
}

\author{
Emmanuelle Waubant ${ }^{1}$
}

Received: 23 January 2021 / Accepted: 30 April 2021/Published online: 25 May 2021

(C) Center for Excellence in Brain Science and Intelligence Technology, Chinese Academy of Sciences 2021

In this issue, the publication of Xiu et al. [1] provides novel and important information on the incidence and outcomes of acute disseminated encephalomyelitis (ADEM) nationwide in China. The study analyzed information from 2,976 cases of ADEM that occurred over a 3-year period, and confirms ADEM is much less common than diseases such as multiple sclerosis (MS) and neuromyelitis optica spectrum disorders.

This excellent work represents the largest study done in the field of ADEM, leveraging an extensive network of tertiary hospitals $(n=1,665)$ using the same electronic medical record system and disease definitions [2]. It provides precious data on ADEM, a relatively rare disease thought to be caused by an immune attack directed against the central nervous system (CNS) in response to infections.

Although ADEM is typically a monophasic disease, it can result in significant morbidity and the causes associated with mortality have been scantly studied. This is why understanding ADEM incidence and the length and cost of hospital stay according to age add very valuable information to capture the yearly disease burden in a single country. Most prior publications have focused on ADEM incidence in a limited region or age range [3]. Using the same methodology for all ages, the authors show a clear predominance in children compared to adults, with the incidence more than 3 times higher in children. This striking difference raises questions regarding the underlying biological processes leading to this post-infectious complication which is less frequent with age [4]. Are the

Emmanuelle Waubant

Emmanuelle.Waubant@ucsf.edu

1 Department of Neurology, University of California at San Francisco, San Francisco, CA, USA same viruses underpinning ADEM in children and adults? Is a mature brain less prone to a post-infectious immune attack or is a more mature immune response less likely to result in a CNS-based attack? We also cannot rule out that a sizeable proportion of those ADEM cases may in fact have been related to myelin oligodendrocyte glycoprotein antibodies as reported in children [5].

The study highlights that the duration of hospital stay for ADEM was pretty long (15 to 21 days on average) and was longer in adult patients with a higher proportion of adults dying from the disease compared to children. Leading causes of death included lung infection and acute respiratory failure, but in children death from cerebral edema was more common than in adults. Encouraging trends include the progressive decrease in mortality over the 3 years encompassed by the study, probably due to earlier diagnosis and improved management.

Because China is such a large country, the study also provides very interesting data on regional incidence in areas with very different climates, population density and life-style, and genetic make-up. In fact, the study reports no statistical difference across the various regions. Furthermore, the incidence of ADEM reported in this work is similar to several other countries with very different climate and genetic make-up. What does this tell us regarding the etiology of the disease? Are the rates of circulation of viruses implicated in ADEM onset similar all over the world? Or are different viruses involved in different regions of the world? Several autoimmune diseases, such as multiple sclerosis, do have marked incidence differences according to climate and genetic make-up of the population. Apparently, not ADEM. These epidemiological findings can help shape how we should think about possible biological processes contributing to disease onset. 
For the first time, the study provides the incidence of ADEM across all age groups in Chinese in mainland of China. The precedent of such an extensive epidemiological study on such a huge population is seldomly set prior to this study. It fills the gaps in epidemiologic data of the approximately 1.4 billion population of China and enriches the global map of this disease. Also, the study overviews the length and cost of hospital stay in China calling for increasing efforts on ADEM patients care and management. The novelty and importance of this study will gain growing interest of global readership of patients and their families, neurologists, neuroscientists and health care policy makers. Moving forward, studies will need to pay continuous attention on ADEM incidence in China seeking clues for further ADEM research.

Conflict of interest The author declare that there is no conflict of interest.

\section{References}

1. Xiu Y, Gu H, Li X, Li Z, Jin WN, Liu Q. Incidence and mortality of acute disseminated encephalomyelitis in China: A nationwide population-based study. Neurosci Bull 2021, https://doi.org/10. 1007/s12264-021-00642-7.

2. Krupp LB, Tardieu M, Amato MP, Banwell B, Chitnis T, Dale RC, et al. International Pediatric Multiple Sclerosis Study Group criteria for pediatric multiple sclerosis and immune-mediated central nervous system demyelinating disorders: revisions to the 2007 definitions. Mult Scler 2013, 19: 1261-1267.

3. Bhatt P, Bray L, Raju S, Dapaah-Siakwan F, Patel A, Chaudhari R, et al. Temporal trends of pediatric hospitalizations with acute disseminated encephalomyelitis in the united states: An analysis from 2006 to 2014 using national inpatient sample. J Pediatr 2019, 206: $26-32$ e21.

4. Cole J, Evans E, Mwangi M, Mar S. Acute disseminated encephalomyelitis in children: an updated review based on current diagnostic criteria. Pediatr Neurol 2019, 100: 26-34.

5. Waters P, Fadda G, Woodhall M, O’Mahony J, Brown RA, Castro DA, et al. Serial anti-myelin oligodendrocyte glycoprotein antibody analyses and outcomes in children with demyelinating syndromes. JAMA Neurol 2020, 77: 82-93. 